\title{
LARGE TIME BEHAVIOR OF PERIODIC VISCOSITY SOLUTIONS FOR UNIFORMLY ELLIPTIC INTEGRO-DIFFERENTIAL EQUATIONS
}

\author{
GUY BARLES, EMMANUEL CHASSEIGNE, ADINA CIOMAGA, AND CYRIL IMBERT
}

\begin{abstract}
In this paper, we study the large time behavior of solutions of a class of parabolic fully nonlinear integro-differential equations in a periodic setting. In order to do so, we first solve the ergodic problem (or cell problem), i.e. we construct solutions of the form $\lambda t+v(x)$. We then prove that solutions of the Cauchy problem look like those specific solutions as time goes to infinity. We face two key difficulties to carry out this classical program: (i) the fact that we handle the case of "mixed operators" for which the required ellipticity comes from a combination of the properties of the local and nonlocal terms and (ii) the treatment of the superlinear case (in the gradient variable). Lipschitz estimates previously proved by the authors (2012) and Strong Maximum principles proved by the third author (2012) play a crucial role in the analysis.
\end{abstract}

\section{INTRODUCTION}

In this paper, we provide new results on the large time behavior of viscosity solutions for parabolic integro-differential equations (PIDE in short).

1.1. A model equation. In order to describe our approach and our results, we consider the following model example

$$
\partial_{t} u-\Delta_{x_{1}} u+(-\Delta)_{x_{2}}^{\beta} u+|D u|^{m}+b_{1}\left(x_{1}\right)\left|D_{x_{1}} u\right|+b_{2}\left(x_{2}\right)\left|D_{x_{2}} u\right|=f(x)
$$

where $u: \mathbb{R}^{d} \times[0,+\infty) \rightarrow \mathbb{R}$ is the unknown function depending on $x=\left(x_{1}, x_{2}\right) \in$ $\mathbb{R}^{d}$ with $x_{1} \in \mathbb{R}^{d_{1}}, x_{2} \in \mathbb{R}^{d_{2}}, d_{1}+d_{2}=d$, and $t \geq 0$. $\partial_{t} u$ denotes the derivative of $u$ with respect to $t$, while $D u=\left(D_{x_{1}} u, D_{x_{2}} u\right)$ stands for its gradient with respect to the space variable $x$. The operator $\Delta_{x_{1}} u$ is the usual Laplacian with respect to the $x_{1}$-variable, while $(-\Delta)_{x_{2}}^{\beta} u$ denotes the fractional Laplacian of exponent $\beta \in(1,2)$ with respect to the $x_{2}$-variable

$(-\Delta)_{x_{2}}^{\beta} u(x, t)=\int_{z_{2} \in \mathbb{R}^{d_{2}}}\left(u\left(x_{1}, x_{2}+z_{2}, t\right)-u(x, t)-D_{x_{2}} u(x, t) \cdot z_{2} 1_{B^{d_{2}}}\left(z_{2}\right)\right) \frac{d z_{2}}{\left|z_{2}\right|^{d_{2}+\beta}}$,

where $D_{x_{2}} u$ is the gradient of $u$ with respect to the $x_{2}$-variable and $B^{d_{2}}$ is the unit ball in $\mathbb{R}^{d_{2}}$. Finally, we assume that $m \geq 1$ and $f, b_{i}(i=1,2)$ are realvalued, Lipschitz continuous functions which are respectively $\mathbb{Z}^{d}$ - and $\mathbb{Z}^{d_{i}}$ - periodic. Because of this last assumption, the solution is expected to be $\mathbb{Z}^{d}$-periodic if the initial datum is.

Date: September 8, 2018.

2010 Mathematics Subject Classification. 35B40, 35R09, 35D40, 35D10.

Key words and phrases. Parabolic nonlinear integro-differential equations, Ergodic problem, Long time behavior, Strong maximum principle, Lipschitz estimates. 
1.2. Aim. For such a PIDE, our aim is to show that, for large times $(t \rightarrow+\infty)$, the solution $u$ asymptotically behaves like $\lambda t+v(x)$ where $(\lambda, v) \in \mathbb{R} \times C^{0}\left(\mathbb{R}^{d} ; \mathbb{R}\right)$ is a solution of the associated ergodic (or additive eigenvalue) problem which, for (11), reads

$$
-\Delta_{x_{1}} v+(-\Delta)_{x_{2}}^{\beta} v+|D v|^{m}+b_{1}\left(x_{1}\right)\left|D_{x_{1}} v\right|+b_{2}\left(x_{2}\right)\left|D_{x_{2}} v\right|=f(x)-\lambda .
$$

A key result in this direction is that there exists a unique $\lambda \in \mathbb{R}$ such that this ergodic problem has a periodic (Lipschitz) continuous solution $v$. With such a result in hand, one has to prove the convergence, namely that for every solution $u$ of (1), there exists a solution $(\lambda, v)$ of the ergodic problem such that $u(x, t)-(\lambda t+v(x)) \rightarrow 0$ as $t \rightarrow+\infty$, uniformly in $x \in \mathbb{R}^{d}$.

To do so, we follow a by-now rather classical method which was systematically developed in [14. To carry out this method, the two key ingredients are estimates on the modulus of continuity (Lipschitz estimates in our case), and a Strong Maximum Principle, both for equations (11) and (2).

These needed results were obtained in previous papers. Lipschitz and Hölder estimates were obtained in [4] where an emphasis was made on "mixed operators", i.e. on equations like (11) where the "uniform ellipticity" comes from both integral and differential terms, namely the $\Delta_{x_{1}}$ and $(-\Delta)_{x_{2}}^{\beta}$ terms. This particular form of the equation creates also difficulties for the Strong Maximum (or Comparison) Principle, see [17.

In addition to this specific difficulty coming from "mixed operators", we also want to handle the "superlinear case", namely the case when $m>1$ in (1). This requires additional work and ideas both for solving (2) and for the convergence proof since one needs Lipschitz estimates to "linearize" this term. For this reason, we distinguish two cases: a sublinear and a superlinear one, with respect to the gradient growth.

In the sublinear case the estimates on the modulus of continuity come from the ellipticity of the equation. Indeed, as shown in 4, even though the equation is completely degenerate in the local term and in the nonlocal term, their combination render the diffusion uniformly elliptic. In the superlinear case, although most of these estimates are derived through the same type of arguments under suitable structure conditions on the nonlinearities, there are some situations where they come from the gradient term (cf. the proof of Lemma 1).

It is worth pointing out that some of our results (in particular in the sublinear case) could be proved in an easier way since they do not require such Lipschitz estimates but we have chosen to systematically use them in order to unify the paper and to keep it with a reasonable length.

1.3. The general framework. Let us now present the general framework of our analysis. Although not the most general one, we have chosen this framework since it carries the key difficulties. Extensions to a larger framework are given in Section 5 . We consider parabolic PIDE of the form

$$
\begin{aligned}
& \partial_{t} u+F_{1}\left(x_{1}, D_{x_{1}} u, D_{x_{1} x_{1}}^{2} u, \mathcal{I}_{x_{1}}[x, u]\right) \\
& \quad+F_{2}\left(x_{2}, D_{x_{2}} u, D_{x_{2} x_{2}}^{2} u, \mathcal{I}_{x_{2}}[x, u]\right)+H(D u)=f(x), \quad \text { in } \mathbb{R}^{d} \times(0,+\infty)
\end{aligned}
$$

(where $D_{x_{i} x_{i}}^{2} u$ denotes the Hessian matrix with respect to $x_{i}, i=1,2$ ) subject to the initial condition

$$
u(x, 0)=u_{0}(x), \quad \text { in } \mathbb{R}^{d}
$$


where $u_{0}$ and $f$ are $\mathbb{Z}^{d}$-periodic functions, $H$ is a continuous function in $\mathbb{R}^{d}$ and $F_{1}, F_{2}$ are nonlinear "elliptic" terms (precise assumptions are given in Section 2). We point out that each nonlinear term involves second-order derivatives and a nonlocal operator of "order" $\beta \in(1,2)$. These operators $\mathcal{I}_{x_{i}}$ are of Lévy-Itô type: if $\varphi: \mathbb{R}^{d_{i}} \rightarrow \mathbb{R}$ is a smooth bounded function, then

$$
\mathcal{I}_{i}[\varphi]\left(x_{i}\right)=\int_{z_{i} \in \mathbb{R}^{d_{i}}}\left(\varphi\left(x_{i}+j_{i}\left(x_{i}, z_{i}\right)\right)-\varphi\left(x_{i}\right)-D \varphi\left(x_{i}\right) \cdot j_{i}\left(x_{i}, z_{i}\right) 1_{B^{d_{i}}}\left(z_{i}\right)\right) d \mu_{i}\left(z_{i}\right)
$$

where $j_{i}$ are the jump functions and $\mu_{i}$ the Lévy measures (here we also refer the reader to Section 2 for precise assumptions). Then we set

$$
\mathcal{I}_{x_{1}}[x, u]:=\mathcal{I}_{1}\left[u\left(\cdot, x_{2}, t\right)\right]\left(x_{1}\right) \quad \text { and } \quad \mathcal{I}_{x_{2}}[x, u]:=\mathcal{I}_{2}\left[u\left(x_{1}, \cdot, t\right)\right]\left(x_{2}\right) .
$$

We recall that these operators are "natural" generalizations of diffusion operators of the form $\frac{1}{2} \operatorname{Tr}\left(\sigma(x) \sigma^{T}(x) D^{2} u\right)$ where $\sigma$ is the diffusion matrix and $\sigma^{T}$ denotes its transpose; in particular, they can be characterized as the infinitesimal generators of some solutions of stochastic differential equations driven by a general Lévy process, instead of a Brownian motion; see 1 for more details.

In the case of (1), we have

$$
\begin{aligned}
F_{1}\left(x_{1}, D_{x_{1}} u, D_{x_{1} x_{1}}^{2} u, \mathcal{I}_{x_{1}}[x, u]\right) & :=-\Delta_{x_{1}} u+b_{1}\left(x_{1}\right)\left|D_{x_{1}} u\right|, \\
F_{2}\left(x_{2}, D_{x_{2}} u, D_{x_{2} x_{2}}^{2} u, \mathcal{I}_{x_{2}}[x, u]\right) & :=(-\Delta)_{x_{2}}^{\beta} u+b_{2}\left(x_{2}\right)\left|D_{x_{2}} u\right|, \\
H(D u) & :=|D u|^{m} .
\end{aligned}
$$

By choosing such a framework, we want to shed some light on the fact that each nonlinear term can have different forms of (local or nonlocal) diffusions in the different sets of variables while the $H$-term carries the possible super-linearity (see Assumptions (H-a) and (H-b) below). Of course this simplified framework can be generalized and we refer the reader to Section 5 devoted to the extensions to see how this can be done for both the $H$-term but also for $F_{1}$ and $F_{2}$.

1.4. Known results. The study of large time behavior of solutions of HamiltonJacobi and fully nonlinear parabolic equations has attracted a lot of attention, in different contexts. It follows closely the development of the viscosity solution theory. Indeed, the first result one could cite about the large time behavior of solutions of first-order $x$ independent equations is already contained in [33] (see also [3]).

When studying the large time behavior of solutions of such equations, one has first to construct solutions of the form $\lambda t+v(x)$; equivalently, one has to solve a stationary equation depending on a parameter $\lambda$ which is unknown. This is the so-called cell (or ergodic) problem. Then one has to prove that the solution of the Cauchy problem indeed converges towards $\lambda t+v(x)$ for some solution $(\lambda, v)$ of the cell problem. The first step was completed in the seminal (yet unpublished) work of Lions, Papanicolaou and Varadhan 34 about homogenization of first-order Hamilton-Jacobi equations (in the case of coercive Hamiltonians). Indeed, solving the cell problem is the way the "effective" (or averaged) Hamilton-Jacobi Equation is determined.

The second step was first completed, for Hamilton-Jacobi Equations, by Namah and Roquejoffre 37. for equations with a particular structures and then by Fathi 21] in the general case of convex and coercive Hamilton-Jacobi Equations in the periodic setting. It is worth pointing out that the proof of [37] is based on pde 
methods while the results of 21] relies on dynamical systems arguments ("Weak KAM method"). Afterwards J.-M. Roquejoffre [39] and A. Davini and A. Siconolfi in [19] refined the approach of A. Fathi and they studied the asymptotic problem for Hamilton-Jacobi Equations on a compact manifold.

Barles and Souganidis extended in [12] the previous results and showed the asymptotic behavior under weaker convexity assumptions, using viscosity solutions techniques. They also gave counterexamples in 13 on the asymptotic behavior of solutions of Hamilton-Jacobi Equations, when the initial datum is not periodic anymore. Motivated by the latest works, Ishii established in 31] a general convergence result for Hamilton-Jacobi equations on the whole space. For asymptotic behavior of solutions of various boundary value problems for Hamilton-Jacobi Equations we refer to the works $28,32,35,10,26,9$, based either on weak KAM theory or on PDEs techniques. Lastly, we mention that Dirr and Souganidis showed in 20] that the asymptotic behavior remains true if one perturbes with additive noise viscous or non-viscous Hamilton-Jacobi equations, periodic in space.

As we already mentioned, our approach follows the ideas systematically developed by Barles and Souganidis in [14, where they described the long time behavior of space-time periodic solutions of quasilinear PDEs. This behavior has also been established for semilinear equations, with methods of degree theory, by Namah and Roquejoffre in 36. Long time behavior and ergodic problems for second order PDEs with Neumann boundary conditions have been studied in the series of papers [6, 18, 17. Recently, viscous Hamilton-Jacobi equations have been treated by Tchamba in the superquadratic case [41, and Barles, Porretta and Tchamba in the subquadratic case [11.

We already mentioned that, when studying homogenization of Hamilton-Jacobi or fully nonlinear elliptic equation, it is necessary to solve a cell problem. As far as nonlinear integro-differential equations with periodic data are concerned, we can first mention a series of papers devoted to the homogenization of dislocation dynamics, see e.g. [30, 22. Some results were also obtained for linear integrodifferential equations with periodic data in [16, 2] by analytical methods and in 42, 25, 23, 24 by probabilistic ones. Homogenization of Markov processes governed by certain Levy operators was discussed by Horie Inuzuka and Tanaka in 27] and general results on their convergence in law were established in [25] (see also [42]). In 23, the author studies the convergence in law of a rescaled solution of a stochastic differential equation driven by an $\beta$-stable Lévy process, $\beta>1$. The author points out that such an homogenization question was raised in [15. p. 531]. It is also mentioned in the introduction of [38, that they are very few such results.

For nonlinear equations, Schwab [40] established homogenization results for a large class of equations. In the previously mentioned papers, both in the linear and the nonlinear cases, an ergodic problem has to be solved. In [23, since the equation is linear, linear equation techniques are used such as the study of the resolvent. In papers such as [30, 22, 2, 40, viscosity solutions / maximum principle techniques are used.

1.5. Organization of the article. The paper is organized as follows. In Section2 assumptions on the nonlocal operator $\mathcal{I}$ and the nonlinearities $H, F_{1}, F_{2}$ are given. We also point out which known results from [8, 17, 4] can be used with such a set of assumptions. In Section 3 we solve the stationary ergodic problem. In Section 4 we state and prove the convergence result for solutions of the Cauchy problem. In 
Section [5] we explain how to extend the previous results to even more equations. In Section 6, we give examples of applications of our results on specific equations.

\section{Assumptions}

Before stating our assumptions, we want to point out that, in order to avoid too many technicalities, we are going to use simplified assumptions for the main results, in particular some strong assumptions on the homogeneity of $F_{1}$ and $F_{2}$. Then in Section 5 we explain how to extend our results to more general situations, under weaker homogeneity and growth assumptions.

Thus, the set of hypotheses below may not always seem consistent when considered as a whole. Typically, assuming the homogeneity assumption (F0) would simplify the general growth hypothesis (F2) a lot. But we keep these assumptions as such since in the extension section, we shall use some of them in their general versions.

We begin with assumptions on the singular measures and jump functions.

(M1) (Integrability) For $i=1,2, \mu_{i}$ is a Lévy measure, i.e. there exists $\tilde{C}_{\mu}>0$ such that,

$$
\int_{\mathbb{R}^{d_{i}}} \min \left(\left|z_{i}\right|^{2}, 1\right) d \mu_{i}\left(z_{i}\right) \leq \tilde{C}_{\mu} \quad(\text { for } i=1,2)
$$

(M2) (Regularity of the measures) There exists $\bar{C}_{\mu}$ and, for $i=1,2$, there exists $\beta_{i} \in(1,2)$ such that, for $\delta>0$ small enough

$$
\int_{B^{d_{i}} \backslash B_{\delta}^{d_{i}}}\left|z_{i}\right| d \mu_{i}\left(z_{i}\right) \leq \bar{C}_{\mu} \delta^{1-\beta_{i}}
$$

where $B_{\delta}^{d_{i}}$ is the ball centered at $0 \in \mathbb{R}^{d_{i}}$ and of radius $\delta$.

(M3) (Jump size) There exist two constants $c_{0}, C_{0}>0$ such that, for any $x \in \mathbb{R}^{d}$ and $i=1,2$

$$
c_{0}\left|z_{i}\right| \leq\left|j_{i}\left(x_{i}, z_{i}\right)\right| \leq C_{0}\left|z_{i}\right|, \quad \text { for all } z_{i} \in B^{d_{i}} .
$$

(M4) (Regularity of the jumps) There exist a constant $\tilde{C}_{0}>0$ such that, for any $x_{i}, y_{i} \in \mathbb{R}^{d_{i}}$

$$
\left|j_{i}\left(x_{i}, z_{i}\right)-j_{i}\left(y_{i}, z_{i}\right)\right| \leq \begin{cases}\tilde{C}_{0}\left|z_{i}\right|\left|x_{i}-y_{i}\right|, & \text { for all } z_{i} \in B^{d_{i}} \\ \tilde{C}_{0}\left|x_{i}-y_{i}\right|, & \text { for all } z_{i} \in \mathbb{R}^{d_{i}} \backslash B^{d_{i}} .\end{cases}
$$

(M5) (Nondegeneracy) There exists a constant $C_{\mu}>0$ and for $i=1,2$, there exist $\beta_{i} \in(1,2)$ such that, for every $p_{i} \in \mathbb{R}^{d_{i}}$, there exist $0<\eta_{i}<1$ such that the following holds for any $x_{i} \in \mathbb{R}^{d_{i}}$ and $\delta>0$

$$
\int_{\mathcal{C}_{\eta_{i}, \delta}^{i}\left(p_{i}\right)}\left|j_{i}\left(x_{i}, z_{i}\right)\right|^{2} d \mu_{i}\left(z_{i}\right) \geq C_{\mu} \eta_{i}^{\frac{d_{i}-1}{2}} \delta^{2-\beta_{i}}
$$

with

$$
\mathcal{C}_{\eta_{i}, \delta}^{i}\left(p_{i}\right):=\left\{z_{i} \in \mathbb{R}^{d_{i}} ;\left|j_{i}\left(x_{i}, z_{i}\right)\right| \leq \delta,\left(1-\eta_{i}\right)\left|j_{i}\left(x_{i}, z_{i}\right)\right|\left|p_{i}\right| \leq\left|p_{i} \cdot j_{i}\left(x_{i}, z_{i}\right)\right|\right\} .
$$

We point out that these assumptions on the measures and jumps are either classical or used in [8, 4, 17, to obtain uniqueness, regularity results and Strong Maximum/Comparison Principle. 
Now we turn to the assumptions on the nonlinearities $F_{i}, i=1,2, H$ and the source term $f$. Since these assumptions are the same for $F_{1}, F_{2}$, we write them for a general $F$ and in $\mathbb{R}^{\tilde{d}}$, having in mind that they hold for $i=1,2, F=F_{i}$ with $\tilde{d}=d_{i}$. We denote by $\mathbb{S}_{\tilde{d}}$ the space of $\tilde{d} \times \tilde{d}$ symmetric matrices.

(F0) (Homogeneity) For any $\lambda>0, x, p \in \mathbb{R}^{\tilde{d}}, X \in \mathbb{S}_{\tilde{d}}, l \in \mathbb{R}$, we have

$$
F(x, \lambda p, \lambda X, \lambda l)=\lambda F(x, p, X, l) .
$$

(F1) (Periodicity-Continuity) $f$ and $x \mapsto F(x, \cdot, \cdot, \cdot)$ are continuous and $\mathbb{Z}^{\tilde{d}_{-}}$ periodic in $\mathbb{R}^{d}$.

(F2) (Ellipticity-Growth conditions) There exist two bounded functions $\Lambda_{1}, \Lambda_{2}$ : $\mathbb{R}^{\tilde{d}} \rightarrow[0, \infty)$ and a constant $\Lambda_{0}>0$ such that $\Lambda_{1}(x)+\Lambda_{1}(x) \geq \Lambda_{0}$ and some constants $k \geq 0, \tau \in(0,1]$, such that for any $x, y \in \mathbb{R}^{\tilde{d}}, p \in \mathbb{R}^{\tilde{d}}, l \leq l^{\prime}$ and any $\varepsilon>0$

$$
\begin{aligned}
F\left(y, p, Y, l^{\prime}\right)- & F(x, p, X, l) \leq \\
& \Lambda_{1}(x)\left(\left(l-l^{\prime}\right)+\frac{|x-y|^{2}}{\varepsilon}+|x-y|^{\tau}|p|^{k+\tau}+C_{1}|p|^{k}\right)+ \\
& \Lambda_{2}(x)\left(\operatorname{Tr}(X-Y)+\frac{|x-y|^{2}}{\varepsilon}+|x-y|^{\tau}|p|^{2+\tau}+C_{2}|p|^{2}\right)
\end{aligned}
$$

if $X, Y \in \mathbb{S}_{\tilde{d}}$ satisfy the inequality

$$
-\frac{1}{\varepsilon}\left[\begin{array}{cc}
I & 0 \\
0 & I
\end{array}\right] \leq\left[\begin{array}{cc}
X & 0 \\
0 & -Y
\end{array}\right] \leq \frac{1}{\varepsilon}\left[\begin{array}{cc}
Z & -Z \\
-Z & Z
\end{array}\right]
$$

with $Z=I-\omega \hat{p}_{0} \otimes \hat{p}_{0}$, for some unit vector $\hat{p}_{0} \in \mathbb{R}^{\tilde{d}}$, and $\omega \in(1,2)$.

As we mention it at the beginning of the section, (F2) does not seem to be consistent with (F0), nor will be the next assumption (F3). However, we will comment the more general framework in Section 5 dedicated to extensions. In the sequel, we use the notations $\Lambda_{1}^{i}, \Lambda_{2}^{i}, k^{i}, \tau^{i}$ for the quantities appearing in (F2) when they are related to $F_{i}$.

(F3) (Lipschitz Continuity) $(p, X, l) \mapsto F(x, p, X, l)$ is Lipschitz continuous, uniformly in $x \in \mathbb{R}^{\tilde{d}}$.

(F4) (Regularity) There exists a modulus of continuity $\omega_{F}$ such that for any $\varepsilon>0$

$F\left(y, \frac{x-y}{\varepsilon}, Y, l\right)-F\left(x, \frac{x-y}{\varepsilon}, X, l\right) \leq \omega_{F}\left(\frac{|x-y|^{2}}{\varepsilon}+|x-y|\right)$

for all $x, y \in \mathbb{R}^{\tilde{d}}, X, Y \in \mathbb{S}_{\tilde{d}}$ satisfying the matrix inequality (5) with $Z=I$ and $l \in \mathbb{R}$.

Finally, on the Hamiltonian $H$, we assume one of the two following hypotheses.

(H-a) (Sublinearity) $H$ is locally Lipschitz continuous and there exists a Hamiltonian $\bar{H}(p)$, 1-positively homogeneous such that

$$
\lim _{k \rightarrow \infty} \frac{1}{k} H(k p)=\bar{H}(p) .
$$


(H-b) (Superlinearity) $H$ is locally Lipschitz continuous and there exists $m>1$, $\eta>0, r_{0}>0$ and $0<\mu_{0}<1$ such that, for all $\mu \in\left[\mu_{0}, 1\right]$ and $|p| \geq r_{0}$

$$
\mu H\left(\frac{p}{\mu}\right)-H(p) \geq \eta(1-\mu)|p|^{m} .
$$

We also use below a consequence of (H-b), namely the fact that there exists $\hat{\eta}>0$, $c_{0} \geq 0$ such that, for all $p \in \mathbb{R}^{d}$ and all $c \geq c_{0}$,

$$
c^{-1} H(c p)-H(p) \geq \hat{\eta} c^{m-1}|p|^{m}-(\hat{\eta})^{-1} .
$$

We leave the proof of (6) to the reader: for $|p| \geq r_{0}$, it comes from (H-b) while, for $|p| \leq r_{0}$, it can be deduced from the first case, taking $\hat{\eta}$ small enough.

Comments on the list of assumptions. We need a long list of assumptions in order to apply known results about uniqueness, regularity, Strong Maximum/Comparison Principle etc. for different equations. In order to convince the reader that we can indeed apply all these theorems, we next make a precise list of the ones we will use and we justify that our assumptions imply theirs.

To fit the framework of viscosity solutions (to ensure the existence of continuous solutions when combined with Perron's method, but not only), we will be using the Comparison Principle for both the evolution equation (3) and for the perturbed stationary equation (88) introduced in the next section. Comparison principle has been shown in 8 to hold if a series of assumptions (A1)-(A4) were satisfied. In our case (A1) in [8] comes from (M1), (M3), (M4) in the present paper; (A2) is trivially satisfied; (A3-2) in [8] comes from (F4) in the present paper; (A4) in [8] comes from (F3) in the present paper.

Both for uniqueness of the solution for the ergodic problem corresponding to equation (7) and for establishing the long time behavior of solutions of equation (3) we will be using Strong Comparison Principle of Lipschitz sub- and supersolutions from [17, Theorem 32]; (H) in [17] comes from (F2), (F3) and (H-a)/(H-b) in the present paper. We would like to point out that, from a rigorous point of view, (F3) does not yield $(\mathrm{H})$ in [17. However, in view of the proof of this result, see the very end of it, it is clear that (F3) is enough to conclude.

As mentioned in the introduction, we would like to deal with Lipschitz solutions for the some of the equations that will appear in the proof. Regularity of solutions for Eq. (10), and in particular Lipschitz estimates, follows from [4, Corollary 7]. Indeed, take $F_{0}=\delta u+H(D u)$ which satisfies (H0) and (H2) from [4; (H1) for $F_{1}$ and $F_{2}$ in [4] follows from (F2) in the present paper; (H2) in [4] follows from (F3) in the present paper; (H3) in 4 follows from (F4) in the present paper; (J1)-(J5) in 4 follows from (M1)-(M5) in the present paper. We also need Lipschitz estimates given by [4, Corollary 7] for solutions of equation (11)in the sublinear case. Choose $F_{0}=\delta u$, and replace $F_{1}(x, p, X, l)$ with $F_{1}(p, X, l)+c^{-1} H(c p)$. Then $F_{0}$ trivially satisfies (H0) and (H2) from 44; (H1) for $F_{1}$ in 4 follows from (F3) and (H-a) in the present paper; (M3)-(J4) in 4 follows from (M3)-(M4) in the present paper.

Finally, to solve the ergodic problem in the sublinear case, we will make usage of the Strong Maximum/Comparison Principle from [17, Theorem 20] for Eq. (12). This equation is degenerate elliptic and nonlinearities are continuous, i.e. it satisfies (E) from [17; moreover, it is 1-homogeneous thanks to (F0) and (H-a); in particular, it satisfies the scaling assumption (S) of [17. Thanks to (M5) and (F3), it also satisfies $\left(\mathrm{N}_{\mathrm{LI}}\right)$. 


\section{The STATIONARY ERGODiC PROBLEM}

In this section we discuss the solvability of the stationary ergodic problem. For the sake of simplicity we write below

$$
\begin{aligned}
& F\left(x, D v, D^{2} v, \mathcal{I}[v]\right) \\
& \quad=F_{1}\left(x_{1}, D_{x_{1}} v, D_{x_{1} x_{1}}^{2} v, \mathcal{I}_{x_{1}}[x, v]\right)+F_{2}\left(x_{2}, D_{x_{2}} v, D_{x_{2} x_{2}}^{2} v, \mathcal{I}_{x_{2}}[x, v]\right) .
\end{aligned}
$$

Our result is the following.

Theorem 1. Assume that (M1)-(M5), (F0)-(F4) with $k_{i} \leq \beta_{i}$, and either (H-a) or $(H-b)$ holds. There exists a unique constant $\lambda \in \mathbb{R}$ for which the stationary ergodic problem

$$
F\left(x, D v, D^{2} v, \mathcal{I}[v]\right)+H(D v)=f(x)-\lambda
$$

has a Lipschitz continuous periodic viscosity solution $v: \mathbb{R}^{d} \rightarrow \mathbb{R}$. Moreover, $v$ is the unique Lipschitz continuous solution of (7), up to an additive constant.

Proof. For any $\delta>0$, we consider as in 34] the following approximate equation

$$
\delta v^{\delta}+F\left(x, D v^{\delta}, D^{2} v^{\delta}, \mathcal{I}\left[v^{\delta}\right]\right)+H\left(D v^{\delta}\right)=f(x) \text { in } \mathbb{R}^{d} .
$$

If $M=\|F(\cdot, 0,0,0)\|_{\infty}+|H(0)|+\|f\|_{\infty}$, we notice that $-\delta^{-1} M$ and $\delta^{-1} M$ are respectively sub- and supersolutions of the above approximated equation. Then it follows from Perron's method for integro-differential equations as described for instance in [29], and from the comparison principle (Theorem 3 of [8]) that there exists a unique bounded viscosity solution $v^{\delta}$ which satisfies

$$
-\frac{M}{\delta} \leq v^{\delta}(x) \leq \frac{M}{\delta}, \quad \text { for all } x \in \mathbb{R}^{d} .
$$

Assumptions (M1),(M3),(M4) and (F4) on $F_{i}, j_{i}$ and $\mu_{i}$ for $i=1,2$ imply that there is a comparison result for (8).

By the periodicity of $F$ and $f, v^{\delta}(\cdot)$ and $v^{\delta}(\cdot+z)$ are both solutions of the above problem, for any $z \in \mathbb{Z}^{d}$. Then the uniqueness of the solution implies that they are equal; hence, $v^{\delta}$ is $\mathbb{Z}^{d}$-periodic.

We next consider $\tilde{v}^{\delta}(\cdot)=v^{\delta}(\cdot)-v^{\delta}(0)$. The following proposition states the uniform boundedness of this sequence of normalized functions. It is the crucial technical part of the analysis of the ergodic problem.

Proposition 1. The sequence $\left\{\tilde{v}^{\delta}\right\}_{\delta}$ is uniformly bounded.

The proof of the proposition is postponed. We remark that $\tilde{v}^{\delta}$ satisfies

$$
\delta \tilde{v}^{\delta}+F\left(x, D \tilde{v}^{\delta}, D^{2} \tilde{v}^{\delta}, \mathcal{I}\left[\tilde{v}^{\delta}\right]\right)+H\left(D \tilde{v}^{\delta}\right)=f(x)-\delta v^{\delta}(0), \quad x \in \mathbb{R}^{d} .
$$

We derive from Proposition 1 and results from [4] that $\left\{\tilde{v}^{\delta}\right\}_{\delta}$ is also equi-Lipschitz continuous (at this point, the whole set of assumptions is required). Therefore, we can use Ascoli's Theorem to extract a subsequence $\tilde{v}^{\delta_{n}}$ which converges locally uniformly (and therefore uniformly, because of the periodicity) to a Lipschitz continuous $\mathbb{Z}^{d}$-periodic function $v$. On the other hand, (9) implies that $\delta_{n} v^{\delta_{n}}(0)$ is bounded; hence, up to extracting again a subsequence, we can further assume that $\delta_{n} v^{\delta_{n}}(0) \rightarrow \lambda$. By the (continuous) stability of viscosity solutions of integrodifferential equations, see e.g. [8], we conclude that $v$ is a solution of (7). 
Next we consider two solutions $\left(\lambda_{i}, v_{i}\right), i=1,2$, of the ergodic problem (7). Then $u_{i}(x, t)=\lambda_{i} t+v_{i}(x)$ are two solutions of (3) with the initial condition

$$
u_{i}(x, 0)=v_{i}(x) \quad x \in \mathbb{R}^{d} .
$$

From the comparison principle for (3), we conclude that, for all $t>0$,

$$
u_{i}(x, t) \leq u_{j}(x, t)+\left\|v_{i}-v_{j}\right\|_{\infty} .
$$

Dividing by $t>0$ and letting $t \rightarrow+\infty$, this implies $\lambda_{i} \leq \lambda_{j}$. Since $i$ and $j$ are arbitrary, we conclude that $\lambda_{1}=\lambda_{2}$.

Finally, thanks to (F3), (H-a)/(H-b) and the Lipschitz continuity of $v_{1}$ and $v_{2}$, we can apply the Strong Comparison Principle from [17, Theorem 32] to the Lipschitz solutions $u_{1}$ and $u_{2}$ of (3) and conclude that $u_{1}-u_{2}=v_{1}-v_{2}$ is constant. It is worth pointing out that this step uses in a crucial way the Lipschitz continuity of $v_{1}$ and $v_{2}$ because of the linearization procedure (but which has to be used only in the superlinear case). This completes the proof of Theorem 1 .

Remark 1. Solving the stationary ergodic problem is still possible for some cases when $\beta<1$, as we will point out in some examples, see Section 6 . As a matter of fact, the solutions $\delta v^{\delta}$ would be Hölder continuous and the stationary ergodic problem would have a Hölder continuous solution. In this case, if the uniqueness of the ergodic constant remains true, it is not clear anymore that the solutions of the ergodic problem are unique up to an additive constant. However, it is worth pointing out that the Lipschitz continuity of the solutions of the ergodic problem is needed (in general) in order to prove the asymptotic result for the PIDE.

We now turn to the proof of the proposition. We distinguish the sublinear and the superlinear case.

Proof of Proposition 1 in the sublinear case. We argue by contradiction: we assume that we can find a subsequence, that we still denote by $\left(\tilde{v}^{\delta}\right)_{\delta}$, for which the associated sequence of norms blows up, i.e.

$$
c_{\delta}:=\left\|\tilde{v}^{\delta}\right\|_{\infty} \rightarrow \infty \quad \text { as } \delta \rightarrow 0 .
$$

We next consider

$$
w^{\delta}(x)=\frac{\tilde{v}^{\delta}(x)}{c_{\delta}}
$$

which, by (F0), satisfies

$$
\delta w^{\delta}+F\left(x, D w^{\delta}, D^{2} w^{\delta}, \mathcal{I}\left[w^{\delta}\right]\right)+\frac{1}{c_{\delta}} H\left(c_{\delta} D w^{\delta}\right)=\frac{f(x)-\delta v_{\delta}(0)}{c_{\delta}} .
$$

Since $\left\|w^{\delta}\right\|_{\infty}=1$ for all $\delta>0,\left\{w^{\delta}\right\}_{\delta}$ is equi-Lipschitz continuous by the results of 4 . Hence, we can extract a locally uniformly converging subsequence (hence globally by periodicity); we denote by $w$ the limit which is a $\mathbb{Z}^{d}$-periodic, Lipschitz continuous function with $\|w\|_{\infty}=1$.

By the (continuous) stability result for viscosity solutions, see e.g. [8], Assumption (H-a) implies that $w$ is a solution of

$$
F\left(x, D w, D^{2} w, \mathcal{I}[w]\right)+\bar{H}(D w)=0, \quad \text { in } \mathbb{R}^{d} .
$$

The limiting equation (12) is 1-homogeneous; in particular, it satisfies the scaling assumption $(\mathrm{S})$ of [17. If we can check that it satisfies $\left(\mathrm{N}_{\mathrm{LI}}\right)$ from [17, then 
this will imply that the equation enjoys the Strong Maximum Principle (cf. [17. Theorem 22]).

This gives the contradiction: indeed, on one hand, we know that $\|w\|_{\infty}=1$ and, by the continuity and periodicity of $w$, its maximum/minimum value is attained and equal to \pm 1 . Henceforth, by the Strong Maximum Principle, the function must be constant equal to \pm 1 . On the other hand $w(0)=0$ since $w^{\delta}(0)=0$ for all $\delta$, which is the desired contradiction.

We now show that indeed, assumption $\left(\mathrm{N}_{\mathrm{LI}}\right)$ of [17] is satisfied, and that it results from from (F2) and (M5). Fix $R_{0}>0$; we must check that for all $R \in\left(0, R_{0}\right)$ and for all $c>0$ we have that

$$
\begin{array}{r}
\mathcal{A}(\gamma):=\sum_{i=1,2} F_{i}\left(x_{i}, p_{i}, I-\gamma p_{i} \otimes p_{i}, \tilde{C}_{\mu}-c \gamma \int_{\tilde{\mathcal{C}}_{\eta_{i}, \gamma}^{i}\left(p_{i}\right)}\left|p_{i} \cdot j_{i}\left(x_{i}, z_{i}\right)\right|^{2} d \mu\left(z_{i}\right)\right) \\
+\bar{H}(p) \rightarrow \infty
\end{array}
$$

as $\gamma \rightarrow \infty$, uniformly for $x \in \mathbb{R}^{d}, p \in B_{R} \backslash B_{R / 2}$. Here the constant $\tilde{C}_{\mu}$ is given by (M1) and the cone is slightly different from the one in (M5). Namely, it has the form

$$
\tilde{\mathcal{C}}_{\eta, \gamma}(p)=\{z ;(1-\eta)|p||j(x, z)| \leq|p \cdot j(x, z)| \leq 1 / \gamma\} .
$$

Going back to the original form of the nonlinearity $F=F_{1}+F_{2}+H$ and using the ellipticity - growth assumption (F2) we get the following lower bound for $\mathcal{A}(\gamma)$,

$$
\begin{array}{r}
\mathcal{A}(\gamma) \geq-\sum_{i=1,2}\left(\Lambda_{1}^{i}(x)\left(\tilde{C}_{\mu}-c \gamma \int_{\tilde{\mathcal{C}}_{\eta_{i}, \gamma}\left(p_{i}\right)}\left|p_{i} \cdot j_{i}\left(x_{i}, z_{i}\right)\right|^{2} d \mu\left(z_{i}\right)\right)+\Lambda_{1}^{i}(x) C_{1}^{i}\left|p_{i}\right|^{k_{i}}+\right. \\
\left.\Lambda_{2}^{i}(x) \operatorname{Tr}\left(I_{d_{i}}-\gamma p_{i} \otimes p_{i}\right)+\Lambda_{2}^{i}(x) C_{2}^{i}\left|p_{i}\right|^{2}\right) \\
\quad+F_{1}\left(x_{1}, p_{1}, 0,0\right)+F_{2}\left(x_{2}, p_{2}, 0,0\right)+\bar{H}(p) .
\end{array}
$$

Using now the structure of the cone, and noting that $\mathcal{C}_{\eta_{i},(R \gamma)^{-1}}(p) \subset \tilde{\mathcal{C}}_{\eta, \gamma}(p)$, we get

$$
\begin{array}{r}
\mathcal{A}(\gamma) \geq-\sum_{i=1,2}\left(\Lambda_{1}^{i}(x)\left(\tilde{C}_{\mu}-c \gamma\left(1-\eta_{i}\right)^{2}\left|p_{i}\right|^{2} \int_{\mathcal{C}_{\eta,(R \gamma)}^{i}\left(p_{i}\right)}\left|j_{i}\left(x_{i}, z_{i}\right)\right|^{2} d \mu\left(z_{i}\right)\right)+\right. \\
\left.\Lambda_{2}^{i}(x)\left(d_{i}-\gamma\left|p_{i}\right|^{2}\right)\right)+\mathcal{O}_{\gamma}(1) .
\end{array}
$$

Employing now the nondegeneracy assumption (M5) and setting by

$$
C:=c C_{\mu} \min _{i=1,2}\left(\left(1-\eta_{i}\right)^{2} \eta_{i}^{\frac{d_{i}-1}{2}}\right) R^{\beta_{i}-2}
$$

we further have that

$$
\begin{aligned}
\mathcal{A}(\gamma) & \geq \sum_{i=1,2}\left(\Lambda_{1}^{i}(x) C\left|p_{i}\right|^{2} \gamma^{\beta_{i}-1}+\Lambda_{2}^{i}(x)\left|p_{i}\right|^{2} \gamma\right)+\mathcal{O}_{\gamma}(1) \\
& \geq \sum_{i=1,2} C \Lambda_{0}\left|p_{i}\right|^{2} \gamma^{\beta_{i}-1}+\mathcal{O}_{\gamma}(1) \\
& =C \Lambda_{0}|p|^{2} \gamma^{\beta_{i}-1}+\mathcal{O}_{\gamma}(1)
\end{aligned}
$$

where $\mathcal{O}_{\gamma}(1)$ is bounded when $\gamma \rightarrow \infty$. Therefore $\mathcal{A}(\gamma) \rightarrow \infty$ as $\gamma \rightarrow \infty$ uniformly in $x$, and $R / 2<|p|<R$ and the proof is now complete in the sublinear case. 
Proof of Proposition 1 in the superlinear case. The beginning of the proof in the superlinear case goes along the same lines as in the sublinear case. We assume that $c_{\delta} \rightarrow \infty$ along a subsequence and reach a contradiction. For simplicity we still keep the notation $\delta$ for the subsequence and assume (with no restriction) that $c_{\delta} \geq 1$ for all $\delta>0$. We consider as before the rescaled functions

$$
w^{\delta}(x):=\frac{\tilde{v}^{\delta}(x)}{c_{\delta}}
$$

so that $\left\|w^{\delta}\right\|_{\infty}=1$ for any $\delta>0$. Rewriting the equation with $w^{\delta}$ and using again the 1-positive homogeneity of nonlinearities, cf. (F0), we see that $w^{\delta}$ still satisfies (11). In particular, we get from (H-b) (and more precisely from (66) ) that

$$
\begin{array}{r}
\hat{\eta}\left|D w^{\delta}\right|^{m} \leq-\frac{1}{c_{\delta}^{m-1}}\left(H\left(D w^{\delta}\right)-(\hat{\eta})^{-1}\right)-\frac{1}{c_{\delta}^{m-1}} F\left(x, D w^{\delta}, D^{2} w^{\delta}, \mathcal{I}\left[x, w^{\delta}\right]\right) \\
-\frac{\delta w^{\delta}}{c_{\delta}^{m-1}}+\frac{f(x)-\delta v^{\delta}(0)}{c_{\delta}^{m}} .
\end{array}
$$

We next claim that the following holds true.

Lemma 1. The family $\left\{w^{\delta}\right\}_{\delta>0}$ is equicontinuous in $\mathbb{R}^{d}$.

The proof of the lemma is postponed and we complete the proof of the proposition. Since $\left\|w_{\delta}\right\|_{\infty} \leq 1$, using Ascoli's Theorem, we can extract a subsequence which converges locally uniformly (hence globally) towards a continuous $\mathbb{Z}^{d}$-periodic function $w$. Using standard stability results for viscosity solutions together with Estimate (9), $c_{\delta} \rightarrow \infty$ and (F4), we get

$$
\hat{\eta}|D w|^{m} \leq 0 .
$$

From this we deduce that $D w=0$ in the viscosity sense. But there we get a contradiction from the continuity of $w$ since $w(0)=0$ and $\|w\|_{\infty}=1$. The proof of the proposition is now complete.

Proof of Lemma 1. We first fix a parameter $\alpha \in(0,1)$ and claim that

Claim 1. For any $\mu \in(0,1)$, there exists a (large) constant $L(\mu)>0$ such that for any $x, y \in \mathbb{R}^{d}$, we have

$$
w^{\delta}(x)-w^{\delta}(y) \leq L(\mu)|x-y|^{\alpha}+2(1-\mu) .
$$

It is classical that such a result implies that $w^{\delta}$ is uniformly continuous and that the modulus of continuity only depends on $L$ and $\alpha$. To prove the claim, we consider the function

$$
\Phi(x, y):=\mu w^{\delta}(x)-w^{\delta}(y)-L|x-y|^{\alpha} .
$$

Since $w^{\delta}$ is $\mathbb{Z}^{d}$-periodic, this function reaches its maximum at some point $(\bar{x}, \bar{y})$ that we may consider in the same cell. If this maximum is nonpositive, then we are done since this means that $w^{\delta}(x)-w^{\delta}(y) \leq L|x-y|^{\alpha}$ for any $x, y \in \mathbb{R}^{d}$.

Otherwise there are two options: $(i)$ either $\bar{x}=\bar{y} ;(i i)$ or $\bar{x} \neq \bar{y}$.

In case $(i)$ we have for any $x, y$

$$
\mu w^{\delta}(x)-w^{\delta}(y)-L|x-y|^{\alpha} \leq(\mu-1) w^{\delta}(\bar{x}),
$$

which implies, together with $\left\|w^{\delta}\right\|_{\infty} \leq 1$, that

$$
w^{\delta}(x)-w^{\delta}(y) \leq L|x-y|^{\alpha}+2(1-\mu),
$$


and the claim holds.

In case $(i i)$, we can use the viscosity inequalities since the functions $L|\cdot-\bar{y}|^{\alpha}$ and $L|\bar{x}-\cdot|^{\alpha}$ are smooth near $\bar{x}$ and $\bar{y}$ respectively. Notice that $\max \Phi \geq \Phi(\bar{x}, \bar{x})=$ $(\mu-1) w^{\delta}(\bar{x})$ which gives the estimate

$$
|\bar{x}-\bar{y}| \leq\left(\frac{2}{L}\right)^{1 / \alpha}
$$

The equation for $\mu w^{\delta}$ can be derived from (11)

$$
F\left(\bar{x}, D\left(\mu w^{\delta}\right), D^{2}\left(\mu w^{\delta}\right), \mathcal{I}\left[\bar{x}, \mu w^{\delta}\right]\right)+\frac{H\left(\left(c_{\delta} / \mu\right) D\left(\mu \tilde{w}^{\delta}\right)\right)}{c_{\delta} / \mu}+\delta \mu w^{\delta}=\frac{f(x)-\delta v^{\delta}(0)}{c_{\delta} / \mu} .
$$

We use the nonlocal version of Jensen-Ishii's Lemma (cf. 8]) and computations from [5. p.14] (see Step 2 of the proof of Theorem 3.1 of [5]) in order to get for all $\delta>0$ two matrices $X, Y \in \mathbb{S}_{d}$ such that

$$
\begin{array}{r}
F\left(\bar{x}, P, X, \mathcal{I}\left[\bar{x}, \mu w^{\delta}\right]\right)+\frac{H\left(\left(c_{\delta} / \mu\right) P\right)}{c_{\delta} / \mu}+\delta \mu w^{\delta}(\bar{x}) \leq \frac{f(\bar{x})-\delta v^{\delta}(0)}{c_{\delta} / \mu} \\
F\left(\bar{y}, P, Y, \mathcal{I}\left[\bar{y}, w^{\delta}\right]\right)+\frac{H\left(c_{\delta} P\right)}{c_{\delta}}+\delta w^{\delta}(\bar{y}) \geq \frac{f(\bar{y})-\delta v^{\delta}(0)}{c_{\delta}}
\end{array}
$$

where

$$
P=\alpha L|\bar{x}-\bar{y}|^{\alpha-2}(\bar{x}-\bar{y})
$$

and $X, Y \in \mathbb{S}_{d}$ satisfy

$$
\left[\begin{array}{cc}
X & 0 \\
0 & -Y
\end{array}\right] \leq \frac{2}{\bar{\varepsilon}}\left[\begin{array}{cc}
Z & -Z \\
-Z & Z
\end{array}\right] \leq \frac{6}{\bar{\varepsilon}}\left[\begin{array}{cc}
I & -I \\
-I & I
\end{array}\right]
$$

where $Z=I-(1+\varpi) \hat{a} \otimes \hat{a}$ with $a=\bar{x}-\bar{y}$ and $\bar{\varepsilon}=(L \alpha)^{-1}|\bar{x}-\bar{y}|^{2-\alpha}$ and $\varpi \in(0,1 / 3)$. Now we subtract both inequalities and we obtain

$$
\frac{H\left(\left(c_{\delta} / \mu\right) P\right)}{c_{\delta} / \mu}-\frac{H\left(c_{\delta} P\right)}{c_{\delta}} \leq(1+\mu) \frac{\|f\|_{\infty}+M}{c_{\delta}}+(1+\mu) \delta+T_{\text {h.o.t. }}
$$

where

$$
T_{\text {h.o.t. }}=F\left(\bar{y}, P, Y, \mathcal{I}\left[\bar{y}, w^{\delta}\right]\right)-F\left(\bar{x}, P, X, \mathcal{I}\left[\bar{x}, \mu w^{\delta}\right]\right) .
$$

Using computations from [4, we get the following estimate of the higher order terms.

Lemma 2. There exists $\tilde{c}$ depending on constants appearing in (M1)-(M5) such that

$$
\mathcal{I}\left[\bar{x}, \mu w^{\delta}\right]-\mathcal{I}\left[\bar{y}, w^{\delta}\right] \leq \tilde{c} .
$$

Proof. We use [4, Corollary 16] with $u=\mu w^{\delta}$ and $v=w^{\delta}, t_{0}=2 \sqrt{d}, \gamma=1$. We then get the desired estimate with $\tilde{c}=2\left(C_{\mu}^{2}+C_{\mu}^{3}\right)$ where $C_{\mu}^{2}$ and $C_{\mu}^{3}$ depend on constants appearing in (M1)-(M5). For the precise estimate of $\tilde{c}$ given above, see the proof of [4, Corollary 16]. The proof of Lemma 2 is now complete.

Combining (F3)-(F4) with Lemma 2, we thus get

$$
T_{\text {h.o.t. }} \leq \omega_{F}\left(\varepsilon^{-1}|\bar{x}-\bar{y}|^{2}+|\bar{x}-\bar{y}|\right)+C_{F} \tilde{c}
$$

where $C_{F}$ is the Lipschitz constant coming from (F3) and $\varepsilon=L^{-1}|\bar{x}-\bar{y}|^{2-\alpha}$. We therefore have

$$
T_{\text {h.o.t. }} \leq \omega_{F}\left(L|\bar{x}-\bar{y}|^{\alpha}+|\bar{x}-\bar{y}|\right)+C_{F} \tilde{c} \leq \omega_{F}\left(2+(2 / L)^{1 / \alpha}\right)+C_{F} \tilde{c}
$$


We now turn to first-order terms. We first notice that (13) yields

$$
|P| \geq 2^{\frac{\alpha-1}{\alpha}} \alpha L^{1 / \alpha}
$$

and since we may assume without loss of generality that $c_{\delta} \geq 1$, we have $\left|c_{\delta} P\right| \geq r_{0}$ for $L$ large enough. Therefore we can use (H-b) which yields

$$
\frac{H\left(\left(c_{\delta} / \mu\right) P\right)}{c_{\delta} / \mu}-\frac{H\left(c_{\delta} P\right)}{c_{\delta}} \geq \eta(1-\mu) c_{\delta}^{m-1}|P|^{m} .
$$

Hence (14) together with Lemma 2 yields, for $\delta$ small enough

$$
\eta(1-\mu) c_{\delta}^{m-1}|P|^{m} \leq 2\left(\|f\|_{\infty}+M+1\right)+\omega_{F}\left(2+(2 / L)^{1 / \alpha}\right)+C_{F} \tilde{c}=: \hat{c} .
$$

Now, using the above estimate of $P$ we have

$$
\eta c_{\delta}^{m-1}|P|^{m} \geq c_{0} c_{\delta}^{m-1} L^{m / \alpha}
$$

where $c_{0}=\eta \alpha^{m} 2^{m(\alpha-1 / \alpha)}$. The above inequality then becomes

$$
c_{0}(1-\mu) \leq c_{0} c_{\delta}^{m-1} \leq \hat{c} L^{-m / \alpha} .
$$

Hence, we reach a contradiction by choosing $L$ large enough (depending on $\mu$ ). The proof of the claim (and thus of the lemma) is now complete.

\section{Large Time Behavior}

This section is devoted to the proof of the following theorem.

Theorem 2. For every $\mathbb{Z}^{d}$-periodic, Lipschitz continuous initial data $u_{0}$, the unique $\mathbb{Z}^{d}$-periodic solution $u \in C\left(\mathbb{R}^{d} \times(0,+\infty)\right)$ of (3)-(4) satisfies

$$
u(x, t)-\lambda t-v(x) \rightarrow 0, \quad \text { as } t \rightarrow \infty
$$

where $(\lambda, v)$ is a solution of the stationary ergodic problem (7).

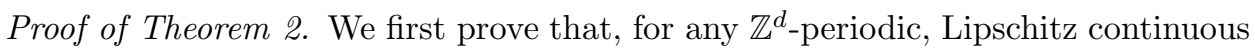
initial data $u_{0}$, Eq. (3)-(44) has a solution $u$. In order to do so, we can assume that $u_{0}$ is $C^{2}$ and bounded, with first and second derivatives bounded as well. Indeed, general initial data $u_{0}$ can be handled thanks to regularization and stability of viscosity solutions of (3), see e.g. 8]. For smooth $u_{0}$ 's, we make the classical observation that $u_{0} \pm C t$ is a super-/subsolution of (3) for $C$ large enough. We thus can apply Perron's method and get a discontinuous solution. The comparison principle for (3) (cf. 8]) then implies that this solution is continuous and unique.

We next follow closely [14. However, we give details for the reader's convenience. The functions $u$ and $\lambda t+v(x)$ are both solutions in $\mathbb{R}^{d} \times(0, \infty)$ of (3). Hence, by the comparison principle for (3), we have

$$
\|u(x, t)-v(x)-\lambda t\|_{\infty} \leq\left\|u_{0}(x)-v(x)\right\|_{\infty}
$$

and, more generally, for all $t \geq s \geq 0$

$$
\max _{x \in \mathbb{R}^{d}}(u(x, t)-\lambda t-v(x)) \leq \max _{x \in \mathbb{R}^{d}}(u(x, s)-\lambda s-v(x)) .
$$

Introducing the notation

$$
m(t)=\max _{x \in \mathbb{R}^{d}}(u(x, t)-\lambda t-v(x)),
$$


we deduce from (15) and (16) that $m(t)$ is nonincreasing and bounded. In particular, there exists $\bar{m} \in \mathbb{R}$ such that

$$
m(t) \searrow \bar{m} \text { as } t \rightarrow \infty
$$

Define next

$$
w(x, t)=u(x, t)-\lambda t .
$$

From (15) we have for all $t>0$,

$$
\|w(\cdot, t)\|_{\infty} \leq\left\|u_{0}-v\right\|_{\infty}+\|v\|_{\infty} .
$$

This $L^{\infty}$-bound implies that $\{w(\cdot, t)\}_{t>0}$ is equi-Lipschitz continuous by the results of [4]. In particular, the sequence $\{w(\cdot, n)\}_{n \in \mathbb{N}}$ is compact in $C\left(\mathbb{R}^{d}\right)$. We thus can extract a converging subsequence $(w(\cdot, \phi(n)))_{n}$

$$
w(x, \phi(n)) \rightarrow \bar{v}(x), \quad x \in \mathbb{R}^{d} .
$$

We next deduce from the comparison principle for (3) that $(w(\cdot, \phi(n)+\cdot))_{n}$ is a Cauchy sequence in $C\left(\mathbb{R}^{d} \times(0, \infty)\right)$. Indeed, for all $t>0$,

$$
\|w(\cdot, t+\phi(n))-w(\cdot, t+\phi(m))\|_{\infty} \leq\|w(\cdot, \phi(n))-w(\cdot, \phi(m))\|_{\infty} .
$$

We thus deduce that $w(x, \phi(n)+t)$ converges uniformly to $\bar{w} \in C\left(\mathbb{R}^{N} \times(0, \infty)\right)$ solving

$$
\begin{cases}\bar{w}_{t}+F\left(x, D \bar{w}, D^{2} \bar{w}, \mathcal{I}[\bar{w}]\right)+H(D \bar{w})+\lambda=f(x) & \text { in } \mathbb{R}^{d} \times(0,+\infty) \\ \bar{w}(x, 0)=\bar{v}(x) & \text { in } \mathbb{R}^{d}\end{cases}
$$

In particular, $\bar{w}(\cdot, t)$ is Lipschitz continuous for all $t>0$. Passing to the limit $\phi(n) \rightarrow \infty$ in

$$
m(\phi(n)+t)=\max _{x \in \mathbb{R}^{d}}(w(x, \phi(n)+t)-v(x))
$$

thanks to the uniform convergence of the sequence $(w(\cdot, \phi(n)+\cdot))_{n}$, we obtain that for all $t>0$,

$$
\bar{m}=\max _{x \in \mathbb{R}^{d}}(\bar{w}(x, t)-v(x)) .
$$

Thanks to (F3), (H2) and the Lipschitz continuity of $\bar{w}(t, \cdot)$ and $v$, we can apply the Strong Comparison Principle from [17]: for all $t>0, x \in \mathbb{R}^{d}$,

$$
\bar{w}(x, t)=v(x)+\bar{m} .
$$

This implies in particular that $\bar{v}=v+\bar{m}$. Hence, $v+\bar{m}$ is the only possible limit of $w(\cdot, n)$; hence $w(x, n) \rightarrow v(x)+\bar{m}$ uniformly as $n \rightarrow \infty$. Now for $t \geq n$, we have

$$
\|u(\cdot, t)-\lambda t-v-\bar{m}\|_{\infty}=\|w(\cdot, t)-v-\bar{m}\|_{\infty} \leq\|w(\cdot, n)-v-\bar{m}\|_{\infty} \rightarrow 0
$$

as $n \rightarrow \infty$. Since the stationary ergodic equation is blind to additive constants, we can replace $v$ with $v+\bar{m}$. The proof is now complete. 


\section{Remarks And Extensions}

In this section, we explain how to study the large time asymptotic of solutions of equations that do not satisfy the assumptions of our main results.

The first key point concerns the existence and uniqueness of viscosity solutions, both for the evolution equation and for the approximate equations which are used to solve the ergodic problem. For this part, (F4) plays a key role and imposes rather strong requirements on the $F_{i}$ 's, which a priori should reduce drastically the growth possibilities in (F2). But one may turn around this difficulty by using a truncation argument: truncating the gradient terms in $F_{1}$ and $F_{2}$ leads to an equation where existence and uniqueness of viscosity solutions holds by assuming only that (F4) holds for bounded $p$ 's, then one can use (F2) to show that the solution is actually Lipschitz continuous (assuming that $u_{0}$ is Lipschitz continuous or using a regularizing effect). In that way, with a slight modification of the formulation of (F4) (which has only to hold for bounded p's), we reconcile Assumptions (F2) and (F4).

But can $F_{1}, F_{2}$ really be superlinear? This question is also connected to the generalization of the $H$-term and we start by this issue.

Following the framework of 4 , one should be able to replace the term $H(D u)$ by $F_{0}\left(D u, D^{2} u, \mathcal{I}[u]\right)$. We recall that, as far as the Lipschitz regularity is concerned, the key point is that this term is independent of $x$ but it can be "degenerate". This is a "neutral" term which does not bring anything positive nor creates any difficulty. The same is true for the comparison result.

For proving Theorem 1 we need the analogue of $(\mathrm{H}-\mathrm{a})-(\mathrm{H}-\mathrm{b})$, i.e. we have to examine $F_{0}(c p, c X, c I)$ as $c$ tends to infinity. The analogous assumptions are

(F0-a) (Sublinearity) $F_{0}$ is locally Lipschitz continuous and there exists a nonlinearity $\overline{F_{0}}, 1$-positively homogeneous such that

$$
\lim _{c \rightarrow \infty} \frac{1}{c} F_{0}(c p, c X, c I)=\overline{F_{0}}(p, X, l) .
$$

(F0-b) (Superlinearity) $F_{0}$ is locally Lipschitz continuous and there exist $m>1$, $\eta>0$ such that, for any $c \geq 1$, for any $p \in \mathbb{R}^{d}, X \in \mathbb{S}_{d}, l \in \mathbb{R}$

$$
\frac{1}{c} F_{0}(c p, c X, c I)-F_{0}(p, X, I) \geq \eta\left(c^{m-1}-1\right)|p|^{m}-\eta^{-1}
$$

This last assumption is obviously satisfied if we add a 1-homogeneous function of $p, X, l$ to an $H$ for which of course the superlinear case may come from $F_{1}, F_{2}$ : typically in the case when $F_{0} \equiv 0$, one may assume that (H-b) holds. On the other hand (F0-b) summarizes the two types of properties we use to prove our results.

We may also assume that $F_{1}, F_{2}$ satisfy (F0-b) with the same $m$ : in this case, the proof follows along the same lines, $|p|^{m}$ being replaced by $\left|p_{1}\right|^{m}+\left|p_{2}\right|^{m}$.

Let us finally mention that our results can be naturally extended to second order fully nonlinear parabolic integro-differential equations, such as those appearing in stochastic control of jump processes. For instance, we can consider the following 
Bellman-Isaacs Equations in $\mathbb{R}^{d}$

$$
\begin{aligned}
\partial_{t} u+\sup _{\gamma \in \Gamma} \inf _{\delta \in \Delta}\left(\begin{array}{rl}
- & \operatorname{tr}\left(A^{\gamma, \delta}(x) D^{2} u\right)-\mathcal{J}^{\gamma, \delta}[x, u]-b^{\gamma, \delta}(x) \cdot D u-f^{\gamma, \delta}(x) \\
& -\frac{1}{2} \operatorname{tr}\left(a_{1}^{\gamma, \delta}\left(x_{1}\right) D_{x_{1} x_{1}}^{2} u\right)-\mathcal{J}_{x_{1}}^{\gamma, \delta}[x, u]-b_{1}^{\gamma, \delta}(x) \cdot D_{x_{1}} u \\
& -\frac{1}{2} \operatorname{tr}\left(a_{2}^{\gamma, \delta}\left(x_{2}\right) D_{x_{2} x_{2}}^{2} u\right)-\mathcal{J}_{x_{2}}^{\gamma, \delta}[x, u]-b_{2}^{\gamma, \delta}(x) \cdot D_{x_{2}} u
\end{array}\right)=0
\end{aligned}
$$

where $\mathcal{J}^{\gamma, \delta}[x, u]$ is a family of Lévy-Itô operators associated with a common Lévy measure $\mu^{0}$ and a family of jump functions $j_{0}^{\gamma, \delta}(x, z)$, respectively $\mathcal{J}_{x_{i}}^{\gamma, \delta}[x, u]$ are families of Lévy-Itô operators associated with the Lévy measures $\mu^{i}$ and the families of jump functions $j_{i}^{\gamma, \delta}\left(x_{i}, z\right)$, for $i=1,2$. We consider that $A^{\gamma, \delta}, a_{i}^{\gamma, \delta}, b_{i}^{\gamma, \delta}, f^{\gamma, \delta}$ are bounded in $W^{1, \infty}$, uniformly in $\gamma$ and $\delta$.

\section{EXAMPLES}

In this section we give several examples for which the previous results (and their extensions from Section (5) apply. We recall that proving this type of behavior required two main tools: regularity of solutions, and Strong Maximum Principle (shortly SMP) for the stationary equation rescaled as in equation (12), and Strong Comparison Principle (shortly SCP) for the evolution equation. We comment three classes of equations: classical diffusions, composed local-nonlocal equations, and mixed equations.

6.1. Classical nonlocal diffusions. Our results apply to a large class of classical PIDE and it would be difficult to illustrate this generality. Therefore, we rather present two particular equations, where we point out some extensions of our results to fractional exponents of lower order $\beta<1$, both in the sublinear and superlinear case.

Example 1 (Fractional diffusions with drift). The model example in this case is the following one

$$
\begin{cases}\partial_{t} u+(-\Delta)^{\beta} u+b(x) \cdot D u=f(x) & \text { in } \mathbb{R}^{d} \times(0, \infty) \\ u(\cdot, 0)=u_{0}(\cdot) & \text { in } \mathbb{R}^{d}\end{cases}
$$

where the vector field $b \in C^{0, \tau}\left(\mathbb{R}^{d} ; \mathbb{R}^{d}\right)$ is $\mathbb{Z}^{d}$-periodic and $u_{0}, f$ are continuous and $\mathbb{Z}^{d}$-periodic. The nonlocal term is a fractional Laplacian of order $\beta \in(1-\tau, 2)$.

$$
(-\Delta)^{\beta} u(x)=-\int_{z \in \mathbb{R}^{d}}\left(u(x+z)-u(x)-D u(x) \cdot z 1_{B^{d}}(z)\right) \frac{d z}{|z|^{d+\beta}} .
$$

Then, for Hölder continuous initial data, the solution is Hölder continuous only if $\beta>1-\tau$. The interesting news in this case is that the corresponding stationary ergodic problem still satisfies SMP, even if $\beta<1$ (by translations of measure supports, see [17]). Hence, by similar arguments, Theorems 1 and 2 hold for fractional exponents if $1-\tau<\beta<2$.

Therefore, the equation has a Hölder continuous viscosity solution $u(x, t)$ which for large times behaves like $\lambda t+v(x)$, with $(\lambda, v) \in \mathbb{R} \times C^{0, \alpha}\left(\mathbb{R}^{d}\right)$ an ergodic pair for

$$
(-\Delta)^{\beta} v+b(x) \cdot D v=f(x)-\lambda \text { in } \mathbb{R}^{d} .
$$


Example 2 (Superlinear Equations). Consider the equation

$$
\begin{cases}\partial_{t} u+(-\Delta)^{\beta} u+b(x)|D u|+|D u|^{m}=f(x) & \text { in } \mathbb{R}^{d} \times(0, \infty) \\ u(\cdot, 0)=u_{0}(\cdot) & \text { in } \mathbb{R}^{d} .\end{cases}
$$

with $u_{0}, f, b \in C^{0, \tau}\left(\mathbb{R}^{d}\right), \beta \in(1-\tau, 2)$, and $m \geq 0$. Here we point out that we may allow $m$ to be less than 1, even if the nonlinearity is not locally Lipschitz continuous because in the linearization process we are using, we can use the Hölder continuity of $p \mapsto|p|^{m}$ instead of its local Lipschitz continuity.

Solutions are Lipschitz continuous for Lipschitz initial data, for all $\beta>1$, and $\tau$ - Hölder continuous for $C^{0, \tau}$ initial data, when $1-\tau<\beta \leq 1$. To establish the long time behavior for any $\beta \in(1-\tau, 2)$ we use as before SMP in the sublinear case $m \leq 1$, and Lemma 1 in the superlinear case $m>1$.

6.2. Composed local-nonlocal diffusion. We refer in the sequel examples to equations which are strictly elliptic in a generalized sense, as introduced in [5: at each point the nonlinearity is either non-degenerate in the second order term, or is non-degenerate in the nonlocal term. One can imagine a partition of the periodic cell in two components such that the local diffusion vanishes on one of them and there the nonlocal term is uniformly elliptic, and vice-versa on the other component.

Example 3. The model example in this case is the following one

$$
\begin{cases}\partial_{t} u-a_{1}(x) \Delta u+a_{2}(x)(-\Delta)^{\beta} u+|D u|^{m}=f(x) & \text { in } \mathbb{R}^{d} \times(0, \infty) \\ u(\cdot, 0)=u_{0}(\cdot) & \text { in } \mathbb{R}^{d} .\end{cases}
$$

with $a_{1}(\cdot)=\sigma^{2}(\cdot), a_{2}(\cdot) \geq 0$, and where $\sigma, a_{2}, f, u_{0}$ are Lipschitz continuous and $\mathbb{Z}^{d}$-periodic. We take $\beta \in(1,2)$, and $m \geq 1$.

Solutions for this equation are Lipschitz continuous when $\beta>1$, provided the two diffusions do not cancel simultaneously

$$
a_{1}(x)+a_{2}(x) \geq a_{0}>0 .
$$

Also, SMP holds (by translations of measure supports) for all $\beta>1$ even if one of the two local or nonlocal diffusion completely degenerates.

However, in order to prove existence of solutions as well as to use SCP, the equation must satisfy hypotheses which ensure comparison/uniqueness results. For this reason, we can either take $a_{2}(x) \equiv a_{2}>0$ for all $x \in \mathbb{R}^{d}$, or we need to impose that $a_{2}(\cdot)^{1 / \beta}$ is Lipschitz continuous, in which case $a_{2}(\cdot)$ can vanish. We emphasize on the fact that in the latter case, we can deal with degenerate nonlocal diffusions and hence work under assumption (19).

Indeed, when $a_{2}(x)>0$ performing the change of variables $z=\alpha(x) z^{\prime}$, with $\alpha(x)=a_{2}(x)^{1 / \beta}$ the nonlocal diffusion can be re-written as

$$
\begin{aligned}
{\left[a_{2}(-\Delta)^{\beta} u\right](x) } & =\int_{\mathbb{R}^{d}}\left(u(x+z)-u(x)-D u(x) \cdot z 1_{B^{d}}(z)\right) \frac{a_{2}(x) d z}{|z|^{d+\beta}} \\
& =\int_{\mathbb{R}^{d}}\left(u(x+\alpha(x) z)-u(x)-D u(x) \cdot \alpha(x) z 1_{B^{d}}(z)\right) \frac{a_{2}(x) \alpha(x)^{d} d z}{\alpha(x)^{d+\beta}|z|^{d+\beta}} .
\end{aligned}
$$

Hence we get a Lévy-Itô operator with jump function $j(x, z)=\alpha(x) z=a_{2}^{1 / \beta}(x) z$

$$
a_{2}(x)(-\Delta)^{\beta} u(x)=\int_{\mathbb{R}^{d}}\left(u\left(x+a_{2}^{1 / \beta}(x) z\right)-u(x)-D u(x) \cdot a_{2}^{1 / \beta}(x) z 1_{B^{d}}(z)\right) \frac{d z}{|z|^{d+\beta}} .
$$


Note that when $a_{2}(x)=0$ for some $x \in \mathbb{R}^{d}$ the above identity remains true.

This nonlocal operator satisfies the hypothesis $(A 1)$ of [8]: the first three integral bounds in $(A 1)$ are immediately satisfied, whereas the last one is true provided that $\beta>1$ and $a_{2}(\cdot)^{1 / \beta}$ is Lipschitz

$$
\begin{aligned}
\int_{\mathbb{R}^{d} \backslash B}|j(x, z)-j(y, z)| \frac{d z}{|z|^{d+\beta}} & =\int_{\mathbb{R}^{d} \backslash B}\left|a_{2}(x)^{1 / \beta}-a_{2}(y)^{1 / \beta}\right||z| \frac{d z}{|z|^{d+\beta}} \\
& \leq \bar{c}_{0}|x-y| \int_{\mathbb{R}^{d} \backslash B}|z| \frac{d z}{|z|^{d+\beta}}=\bar{c}|x-y| .
\end{aligned}
$$

Under this condition, we have the expected asymptotic behavior.

6.3. Mixed local-nonlocal equations. These equations correspond to the so called mixed ellipticity: at each point the nonlinearity is degenerate both in the second order term and the nonlocal term: classical diffusion makes the equation uniformly elliptic in one direction only, say $x_{1}$, and fractional diffusion makes it uniformly elliptic in the complementary direction $x_{2}$. The interesting part is that this combination renders the nonlinearity uniformly elliptic overall. In [17] these equations were proven to satisfy the Strong Maximum and Comparison Principles, and in [4] regularity results were established. We comment below how these two ingredients combine and give the large time behavior. We first consider a toy-model and then a more general equation, so that we can comment precisely the large set of assumptions presented in Section 2 .

Example 4 (Toy model). The model example in this case is the following one

$$
\begin{cases}\partial_{t} u-\Delta_{x_{1}} u+(-\Delta)_{x_{2}}^{\beta} u+|D u|^{m}=f(x) & \text { in } \mathbb{R}^{d} \times(0, \infty) \\ u(\cdot, 0)=u_{0}(\cdot) & \text { in } \mathbb{R}^{d} .\end{cases}
$$

where $u_{0}, f: \mathbb{R}^{d} \rightarrow \mathbb{R}$ are Lipschitz continuous and $\mathbb{Z}^{d}$-periodic, $1<\beta<2, m \geq$ 0 . As before, we point out that we may allow $m$ to be less than 1 , even if the nonlinearity is not locally Lipschitz continuous, because in the linearization process we are using, we can use the Hölder continuity of $p \mapsto|p|^{m}$ instead of its local Lipschitz continuity.

The symbol $\Delta_{x_{1}} u$ stands for the classical Laplacian with respect to $x_{1}$ variable and $(-\Delta)_{x_{2}}^{\beta}$ for the fractional Laplacian of order $\beta$ with respect to $x_{2}$ variable

$$
(-\Delta)_{x_{2}}^{\beta} u(x)=\int_{z \in \mathbb{R}^{d_{2}}}\left(u\left(x_{1}, x_{2}+z\right)-u(x)-D_{x_{2}} u(x) \cdot z 1_{B_{1}^{d_{2}}}(z)\right) \frac{d z}{|z|^{d_{2}+\beta}} .
$$

Then, by the results of [8] and [4, this initial value problem has a unique solution $u(x, t)$ which is Lipschitz continuous in $x$ for all $t \geq 0$, and $\mathbb{Z}^{d}$-periodic. By Theorem 2 the solution asymptotically behaves like

$$
u(x, t)=v(x)+\lambda t+o_{t}(1), \text { as } t \rightarrow \infty,
$$

where $(\lambda, v)$ is a solution of the stationary ergodic problem

$$
-\Delta_{x_{1}} v+(-\Delta)_{x_{2}}^{\beta} v+|D v|=f(x)-\lambda \text { in } \mathbb{R}^{d} .
$$

Solutions of this initial value problem are Hölder continuous for $\beta \in(0,1]$ and Lipschitz continuous for $\beta \in(1,2)$, and any $m \geq 0$. However to solve the stationary problem we either require for the SMP to hold in which case it is necessary that $\beta>1$ and $m \leq 1$ (see [17]), or we can use an estimate as in Lemma 1 to deal with the superlinear case $m>1$. Finally, to establish the long time behavior we need to 
employ SCP: this holds if $m \leq 1$ for Hölder solutions, whereas for $m>1$ it holds only if solutions are Lipschitz continuous, hence $\beta>1$. All in all, the long time behavior is established both in the sublinear and superlinear case, for fractional exponent $\beta>1$.

Example 5 (Mixed equations with first-order-terms). Consider the initial value problem with mixed local-nonlocal diffusion and partial gradient terms

$\left\{\begin{array}{lc}\partial_{t} u-a_{1}\left(x_{1}\right) \Delta_{x_{1}} u-\mathcal{I}_{x_{2}}[u]+b_{1}\left(x_{1}\right)\left|D_{x_{1}} u\right|^{k_{1}}+b_{2}\left(x_{2}\right)\left|D_{x_{2}} u\right|^{k_{2}}=f & \text { in } \mathbb{R}^{d} \times(0, \infty) \\ u(\cdot, 0)=u_{0}(\cdot) & \text { in } \mathbb{R}^{d} .\end{array}\right.$

with $u_{0}, f$ Lipschitz continuous and $\mathbb{Z}^{d}$-periodic, $a_{1}(\cdot)=\sigma^{2}(\cdot) \geq a_{0}$ with $\sigma(\cdot)$ a Lipschitz continuous and $\mathbb{Z}^{d_{1}}$ periodic function, $b_{i} \in C^{0, \tau_{i}}\left(\mathbb{R}^{d_{i}}\right)$ for $\tau_{i} \in(0,1)$ and $\mathbb{Z}^{d_{i}}$ periodic, $i=1,2$. The exponents must satisfy $k_{1} \in\left(0,2+\tau_{1}\right), k_{2} \in\left(0, \beta+\tau_{2}\right)$. The nonlocal operator $\mathcal{I}_{x_{2}}[u]$ is is of Lévy-Itô form, associated to a Lévy measure $\mu$ satisfying (M1)-(M5), with fractional exponent $\beta \in(1,2)$

$$
\mathcal{I}_{x_{2}}[u](x)=\int_{z \in \mathbb{R}^{d_{2}}}\left(u\left(x_{1}, x_{2}+z\right)-u(x)-D_{x_{2}} u(x) \cdot z 1_{B_{1}^{d_{2}}}(z)\right) \mu(d z) .
$$

There exists a solution $u(x, t)$ of this initial value problem which is Lipschitz continuous in space. Once again, by Theorem 2 the solution asymptotically behaves like

$$
u(x, t)=v(x)+\lambda t+o_{t}(1), \text { as } t \rightarrow \infty,
$$

where $(\lambda, v)$ is a solution of the stationary ergodic problem

$$
-a_{1}\left(x_{1}\right) \Delta_{x_{1}} v-\mathcal{I}_{x_{2}}[v]+b_{1}\left(x_{1}\right)\left|D_{x_{1}} v\right|^{k_{1}}+b_{2}\left(x_{2}\right)\left|D_{x_{2}} v\right|^{k_{2}}=f(x)-\lambda \text { in } \mathbb{R}^{d} .
$$

We recall that, in 4, the regularity of solutions is obtained independently for each set of variables $x_{1}, x_{2}$. Roughly speaking, and taking into account the remarks of Section 5 for the superlinear cases, this requires (i) the special form of the equation with no coupling between the $x_{1}$ and $x_{2}$ dependences, and (ii) the $x_{1}$ and $x_{2}$ parts of the equation have both to satisfy hypotheses which ensure Lipschitz regularity results and (iii) comparison results. This justifies the above constraints on $a_{1}, b_{i}, k_{i}, \tau_{i}(\mathrm{i}=1,2)$.

We insist on the fact that we can only deal in the variable $x_{2}$ with Lévy-Itô operators, since uniqueness results were established in [8] for this type of operators only. The general case is still being left as an important open problem. For this reason, we cannot have a coefficient of the form $a_{2}\left(x_{2}\right)$ in front of the nonlocal diffusion $\mathcal{I}_{x_{2}}$ (except for the cases discussed in Example 3), even if, under suitable assumption, we could still have a regularity result.

Thus, one first sees that the solution is Lipschitz continuous with respect to $x_{1}$, for directional gradient terms $b_{1}\left(x_{1}\right)\left|D_{x_{1}} u\right|^{k_{1}}$ having natural growth $k_{1} \leq 2+\tau_{1}$, and Hölder continuous with respect to the $x_{2}$ variable for directional gradient terms $b_{2}\left(x_{2}\right)\left|D_{x_{2}} u\right|^{k_{2}}$ having natural growth $k_{2} \leq \beta+\tau_{2}$. When $\beta>1$, the solution is globally Lipschitz continuous.

For the stationary ergodic problem, there are two cases we can treat: in the sublinear case, namely $k_{1}, k_{2} \leq 1$, we use as before SMP (we refer the reader to Example 4 to see that having Hölder continuous nonlinearities does not create any additional difficulty). In the superlinear case we require $k_{1}, k_{2}>1$ and

$$
b_{1}\left(x_{1}\right), b_{2}\left(x_{2}\right) \geq b_{0}>0 \text { for all } x=\left(x_{1}, x_{2}\right) \in \mathbb{R}^{d} .
$$


There is a slight difficulty to prove Lemma 1 if $k_{1} \neq k_{2}$ : in order to do it, say in the case when $k_{1}<k_{2}$, we divide the equation satisfied by $w^{\delta}$ by $c_{\delta}^{k_{1}-1}$ (as we did it with $c_{\delta}^{m-1}$ for Lemma 1): according to the above assumption on $b_{1}, b_{2}$ the inequality can be written as

$$
b_{0}\left|D_{x_{1}} w^{\delta}\right|^{k_{1}}+b_{0} c_{\delta}^{k_{2}-k_{1}}\left|D_{x_{2}} w^{\delta}\right|^{k_{2}} \leq \cdots,
$$

and since we may assume that $c_{\delta} \geq 1$, we are lead to

$$
b_{0}\left|D_{x_{1}} w^{\delta}\right|^{k_{1}}+b_{0}\left|D_{x_{2}} w^{\delta}\right|^{k_{2}} \leq \cdots \text {. }
$$

This allows to get the same conclusion as in Lemma 1 since (for proving Claim 1) if $D w^{\delta}$ is large, then

$$
b_{0}\left|D w^{\delta}\right|^{k_{1}} \leq 2^{k_{1}}\left(b_{0}\left|D_{x_{1}} w^{\delta}\right|^{k_{1}}+b_{0}\left|D_{x_{2}} w^{\delta}\right|^{k_{2}}\right),
$$

because we have either $\left|D_{x_{1}} w^{\delta}\right| \geq\left|D w^{\delta}\right| / 2$ or $\left|D_{x_{2}} w^{\delta}\right| \geq\left|D w^{\delta}\right| / 2$.

On the other hand, solutions of this equation must satisfy SCP, in order to have the above asymptotic behavior. To this end we need the solutions to be Lipschitz continuous (thus $\beta>1$ ) whenever we deal with $k_{1}, k_{2} \geq 1$.

Example 6 (Sublinear vs. Superlinear Gradients Terms). Consider the initial value problem with mixed local-nonlocal diffusion and partial gradient terms

$\begin{cases}\partial_{t} u-\sigma^{2} \Delta_{x_{1}} u+\mathcal{I}_{x_{2}}[u]+b_{1}\left|D_{x_{1}} u\right|^{k_{1}}+b_{2}\left|D_{x_{2}} u\right|^{k_{2}}+|D u|^{m}=f & \text { in } \mathbb{R}^{d} \times(0, \infty) \\ u(\cdot, 0)=u_{0}(\cdot) & \text { in } \mathbb{R}^{d}\end{cases}$

where $u_{0}, \sigma, f, b_{i}$ and $k_{i}$ are as before. In addition to the previous example we have an extra full gradient term of growth $m \geq 0$.

The above arguments apply here. We just point out the interplay between the different gradient terms. When solving the stationary ergodic problem, we distinguish several different cases. When $m \leq 1$ the equation behaves as in the previous example. Namely either $k_{1}, k_{2} \leq 1$ and the equation is sublinear, thus we apply SMP to conclude as usual; or we require $k_{1}, k_{2}>1$ in which case we have the constraint (20). When $m>1$, we have to distinguish two situations: either $m>\max \left(k_{1}, k_{2}\right)$ and hence the full gradient takes over the other two directional gradients, and in this case $b_{1}(\cdot), b_{2}(\cdot)$ can change sign. Or we can deal with $m<\min \left(k_{1}, k_{2}\right)$ and we argue as in Example 5, under the additional assumption (20).

\section{REFERENCES}

[1] D. Applebaum, Lévy processes and stochastic calculus, vol. 116 of Cambridge Studies in Advanced Mathematics, Cambridge University Press, Cambridge, second ed., 2009.

[2] M. ARISAWA, Homogenization of a class of integro-differential equations with Lévy operators, Comm. Partial Differential Equations, 34 (2009), pp. 617-624.

[3] G. Barles, Asymptotic behavior of viscosity solutions of first Hamilton Jacobi equations, Ricerche Mat., 34 (1985), pp. 227-260.

[4] G. Barles, E. Chasseigne, A. Ciomaga, and C. Imbert, Lipschitz regularity of solutions for mixed integro-differential equations, J. Differential Equations, 252 (2012), pp. 6012-6060.

[5] G. Barles, E. Chasseigne, and C. Imbert, Hölder continuity of solutions of second-order non-linear elliptic integro-differential equations, J. Eur. Math. Soc. (JEMS), 13 (2011), pp. 126.

[6] G. BARles AND F. DA Lio, On the boundary ergodic problem for fully nonlinear equations in bounded domains with general nonlinear Neumann boundary conditions, Ann. Inst. H. Poincaré Anal. Non Linéaire, 22 (2005), pp. 521-541. 
[7] G. Barles, F. Da Lio, P.-L. Lions, and P. E. Souganidis, Ergodic problems and periodic homogenization for fully nonlinear equations in half-space type domains with Neumann boundary conditions, Indiana Univ. Math. J., 57 (2008), pp. 2355-2375.

[8] G. Barles And C. Imbert, Second-order elliptic integro-differential equations: viscosity solutions' theory revisited, Ann. Inst. H. Poincaré Anal. Non Linéaire, 25 (2008), pp. 567585.

[9] G. Barles, H. IshiI, ANd H. Mitake, On the large time behavior of solutions of HamiltonJacobi equations associated with nonlinear boundary conditions, Arch. Ration. Mech. Anal., 204 (2012), pp. 515-558.

[10] G. BARLES AND H. MitAKe, A PDE approach to large-time asymptotics for boundary-value problems for nonconvex Hamilton-Jacobi equations, Comm. Partial Differential Equations, 37 (2012), pp. 136-168.

[11] G. Barles, A. Porretta, And T. T. Tchamba, On the large time behavior of solutions of the Dirichlet problem for subquadratic viscous Hamilton-Jacobi equations, J. Math. Pures Appl. (9), 94 (2010), pp. 497-519.

[12] G. Barles and P. E. Souganidis, On the large time behavior of solutions of Hamilton-Jacobi equations, SIAM J. Math. Anal., 31 (2000), pp. 925-939 (electronic).

[13] G. Barles And P. E. Souganidis, Some counterexamples on the asymptotic behavior of the solutions of Hamilton-Jacobi equations, C. R. Acad. Sci. Paris Sér. I Math., 330 (2000), pp. 963-968.

[14] G. Barles And P. E. Souganidis, Space-time periodic solutions and long-time behavior of solutions to quasi-linear parabolic equations, SIAM J. Math. Anal., 32 (2001), pp. 1311-1323 (electronic).

[15] A. Bensoussan, J.-L. Lions, and G. Papanicolaou, Asymptotic analysis for periodic structures, vol. 5 of Studies in Mathematics and its Applications, North-Holland Publishing Co., Amsterdam, 1978.

[16] E. Chasseigne, M. Chaves, And J. D. Rossi, Asymptotic behavior for nonlocal diffusion equations, J. Math. Pures Appl. (9), 86 (2006), pp. 271-291.

[17] A. Ciomaga, On the strong maximum principle for second order nonlinear parabolic integrodifferential equations, Advances in Differential Equations, 17 (2012), pp. 635-671.

[18] F. DA Lio, Large time behavior of solutions to parabolic equations with Neumann boundary conditions, J. Math. Anal. Appl., 339 (2008), pp. 384-398.

[19] A. Davini and A. Siconolfi, A generalized dynamical approach to the large time behavior of solutions of Hamilton-Jacobi equations, SIAM J. Math. Anal., 38 (2006), pp. 478-502 (electronic).

[20] N. DirR AND P. E. SougAnidis, Large-time behavior for viscous and nonviscous HamiltonJacobi equations forced by additive noise, Siam Journal on Mathematical Analysis, 37 (2005), pp. $777-796$.

[21] A. FAthi, Sur la convergence du semi-groupe de Lax-Oleinik, C. R. Acad. Sci. Paris Sér. I Math., 327 (1998), pp. 267-270.

[22] N. Forcadel, C. Imbert, and R. Monneau, Homogenization of some particle systems with two-body interactions and of the dislocation dynamics, Discrete Contin. Dyn. Syst., 23 (2009), pp. $785-826$.

[23] B. Franke, A functional non-central limit theorem for jump-diffusions with periodic coefficients driven by stable Lévy-noise, J. Theoret. Probab., 20 (2007), pp. 1087-1100.

[24] - Homogenization of random transport along periodic two-dimensional flows, Stochastic Process. Appl., 119 (2009), pp. 327-346.

[25] T. Fujiwara And M. TOMisaki, Martingale approach to limit theorems for jump processes, Stochastics Stochastics Rep., 50 (1994), pp. 35-64.

[26] Y. Giga, Q. LiU, and H. Mitake, Large-time asymptotics for one-dimensional Dirichlet problems for Hamilton-Jacobi equations with noncoercive Hamiltonians, J. Differential Equations, 252 (2012), pp. 1263-1282.

[27] M. Horie, T. InUzuka, And H. TANaka, Homogenization of certain one-dimensional discontinuous Markov processes, Hiroshima Math. J., 7 (1977), pp. 629-641.

[28] N. ICHIHARA AND H. ISHII, Long-time behavior of solutions of Hamilton-Jacobi equations with convex and coercive Hamiltonians, Arch. Ration. Mech. Anal., 194 (2009), pp. 383-419.

[29] C. Imbert, A non-local regularization of first order Hamilton-Jacobi equations, J. Differential Equations, 211 (2005), pp. 218-246. 
[30] C. Imbert, R. Monneau, And E. Rouy, Homogenization of first order equations with $(u / \epsilon)$ periodic Hamiltonians. II. Application to dislocations dynamics, Comm. Partial Differential Equations, 33 (2008), pp. 479-516.

[31] H. IsHII, Asymptotic solutions for large time of Hamilton-Jacobi equations in Euclidean $n$ space, Ann. Inst. H. Poincaré Anal. Non Linéaire, 25 (2008), pp. 231-266.

[32] - Long-time asymptotic solutions of convex Hamilton-Jacobi equations with Neumann type boundary conditions, Calc. Var. Partial Differential Equations, 42 (2011), pp. 189-209.

[33] P.-L. Lions, Generalized solutions of Hamilton-Jacobi equations, vol. 69 of Research Notes in Mathematics, Pitman (Advanced Publishing Program), Boston, Mass., 1982.

[34] P.-L. Lions, G. Papanicolaou, and S. Varadhan, Homogenization of Hamilton-Jacobi equations. Preprint, 1987.

[35] H. Mitake, Asymptotic solutions of Hamilton-Jacobi equations with state constraints, Appl. Math. Optim., 58 (2008), pp. 393-410.

[36] G. Namah And J.-M. Roquejoffre, Convergence to periodic fronts in a class of semilinear parabolic equations, NoDEA Nonlinear Differential Equations Appl., 4 (1997), pp. 521-536.

[37] - Remarks on the long time behaviour of the solutions of Hamilton-Jacobi equations, Comm. Partial Differential Equations, 24 (1999), pp. 883-893.

[38] R. Rhodes and V. Vargas, Scaling limits for symmetric Itô-Lévy processes in random medium, Stochastic Process. Appl., 119 (2009), pp. 4004-4033.

[39] J.-M. Roquejoffre, Convergence to steady states or periodic solutions in a class of Hamilton-Jacobi equations, J. Math. Pures Appl. (9), 80 (2001), pp. 85-104.

[40] R. W. SchwAB, Periodic homogenization for nonlinear integro-differential equations, SIAM J. Math. Anal., 42 (2010), pp. 2652-2680.

[41] T. ТАвет Тснамва, Large time behavior of solutions of viscous Hamilton-Jacobi equations with superquadratic Hamiltonian, Asymptot. Anal., 66 (2010), pp. 161-186.

[42] M. Tomisaki, Homogenization of càdlàg processes, J. Math. Soc. Japan, 44 (1992), pp. 281305.

Laboratoire de Mathématiques et Physique Théorique, CNRS UMR 7350, Fédération Denis Poisson, Université François Rabelais, Parc de Grandmont, 37200 Tours, France

E-mail address: barles@lmpt.univ-tours.fr

Laboratoire de Mathématiques et Physique Théorique, CNRS UMR 7350, Fédération Denis Poisson, Université François Rabelais, Parc de Grandmont, 37200 Tours, France

E-mail address: emmanuel.chasseigne@lmpt.univ-tours.fr

Department of Mathematics, University of Chicago, 5734 University Avenue, ChicaGO, IL 60637, USA

E-mail address: adina@math.uchicago.edu

CNRS, Laboratoire d'Analyse et de Mathématiques Appliquées, UMR 8050, Université Paris-Est Créteil, 61 avenue du Général de Gaulle 94010 Créteil Cedex France

E-mail address: cyril.imbert@u-pec.fr 